\title{
Chemical Etching Treatment of Polydimethylsiloxane for Smoothing Microchannel Surface
}

\author{
Sylvan Sunny Koyagura ${ }^{1}$, Hiroaki Takehara ${ }^{1,2^{*}}$, and Takanori Ichiki ${ }^{1,2^{* * *}}$ \\ ${ }^{1}$ Department of Materials Engineering, School of Engineering, The University of Tokyo, \\ 7-3-1 Hongo, Bunkyo-ku, Tokyo 113-8656, Japan \\ ${ }^{2}$ Innovation Center of NanoMedicine (iCONM), \\ 3-25-14 Tonomachi, Kawasaki, Kanagawa 210-0821, Japan \\ *takehara@bionano.t.u-tokyo.ac.jp \\ **ichiki@bionano.t.u-tokyo.ac.jp
}

\begin{abstract}
This paper investigates the surface modification process of polydimethylsiloxane (PDMS) microchannels for the improvement of the image quality in dark-field imaging with light sheet illumination inside the microchannel. Dark-field light scattering technology in microchannels is a powerful technology used in the visualization of nanoparticles. Wet etching process using Tetrabutylammonium fluoride (TBAF) in $N$-methyl-2-pyrrolidinone (NMP) solvent was used for the surface modification of the PDMS microchannel, and the etch distance of PDMS with $0.68 \mathrm{M}$ of TBAF in NMP solution for 10 min was evaluated as $18.8 \pm 1.8 \mu \mathrm{m}$. Darkfield light scattering imaging of polystyrene nanoparticles $(\phi=100 \mathrm{~nm})$ using light-sheet illumination was demonstrated and the attenuation of scattering light from the microchannel wall was confirmed using the microchannel with TBAF treatment. This surface modification technique improves the imaging quality with light sheet illumination. Keywords: Light sheet technology, Dark field light scattering imaging, Optomicrofluidics, Wet etching process of PDMS, Nanoparticle tracking analysis
\end{abstract}

\section{Introduction}

Surface roughness of microchannel side wall has been focused as one of the important factors in microfluidic devices in such specific applications of heat exchangers and thermal management systems using microfluidic technology [1-5]. The surface roughness of the microchannel side wall often causes fluctuation of flow inside the microchannel and effects convective heat transfer [2]. The such surface roughness of the microchannel wall can be formed in the fabrication process of the microfluidic devices. Soft lithography process is commonly used for the formation of microstructures, such as microchannels [6-9], and the surface roughness of the microchannel wall is due to the master mold, such as Si mold and SU-8 mold in the softlithography process [10].

Polydimethylsiloxane (PDMS) has favorable properties ranging from optical transparency, chemical inertness, ease of handling, and compatibility in the formation of microstructures
$[11,12]$, that makes it suitable for use in optical measurement applications. Recently, light-sheet technology, where only the molecules or particles within the focused plane can be illuminated [13], has been combined with microfluidic devices for planar illumination inside the microchannels [1417]. Dark field light scattering imaging method using the light sheet illumination inside microchannels enables the observation of individual polymer nanoparticles, nanoliposomes and exosomes [18-21]. When considering the surface roughness of the microchannel side wall, the uneven (non-smooth) surface at the interface between PDMS and the sample solution causes refraction and scattering of light sheet and thus, results in the degradation of the image quality due to increase in background noise.

The surface structures of PDMS devices can be modified either using dry or wet etching [22], and wet etching has been reported to be a quicker process than dry etching [23]. Tetrabutylammonium 
fluoride (TBAF) is a prevalent wet etchant that is used to etch PDMS [24-27], for which its mechanism involves the naked fluoride ion in TBAF attacking the siloxane bond in PDMS [26], [28]. Although the effect of the surface roughness on optical property has importance, only a few studies about the surface roughness of the microchannel wall exist and those reported, are in the aspects of effects on fluid flow and thermal transport characteristics.

In this paper, the surface modification process of the PDMS microchannel surface has been investigated for dark-field imaging with light sheet illumination inside the microchannel. The etch distance of PDMS with different concentrations of TBAF solution was evaluated and discussed based on the reaction mechanism of TBAF and PDMS, and the cross-sectional image of the PDMS microchannel was observed using a scanning electron microscope (SEM) to examine the effect of the etching treatment on the shape of the microchannel. Finally, dark field light scattering imaging with light sheet illumination was used for the observation of the individual polymer nanoparticles and the image qualities were compared between treated and untreated TBAF microchannels.

\section{Materials and methods}

\subsection{Photomask fabrication}

A chrome-quartz photomask blank (2.5-inch masks, ULVAC, Inc., Japan) was spin-coated using a spin coater (ACTIVE Spincoater, ACTIVE Co., Ltd., Saitama, Japan) with photoresist (JSR 7790G, JSR Micro NV, Belgium) at a rate of $3000 \mathrm{rpm}$ for $30 \mathrm{sec}$ attaining a resist thickness of $1.1 \mu \mathrm{m}$. Next, pre-bake process was performed at $110^{\circ} \mathrm{C}$ for $90 \mathrm{sec}$ using a hot plate (Digital Hot Plate HP-1SA, AS ONE Corporation, Japan). Figure 1(a) shows a schematic design of a microchannel for dark-field imaging with light sheet illumination. The microchannel design of the photomask was created using Klayout software, and the design was written onto the photomask using a laser lithography system (HEIDELBERG DWL66+, Heidelberg Instruments, Germany). After writing, the photomask was developed for $60 \mathrm{sec}$ using a developer (NMD-W, Tokyo Ohka Kogyo Co., Ltd., Japan) and then rinsed with deionized water. Then, the chrome was etched off the mask using an etchant (Pure Etch CR100, Hayashi Pure Chemical IND. LTD.), and was rinsed with deionized water. Finally, the photoresist was stripped off using ethanol (Ethanol,
Kanto Chemical, Co., Inc., Japan) by an ultrasonication process (Yamato BRANSON 1510, Yamato Scientific Co., Ltd., Japan) to form the fabricated photomask.

(a)

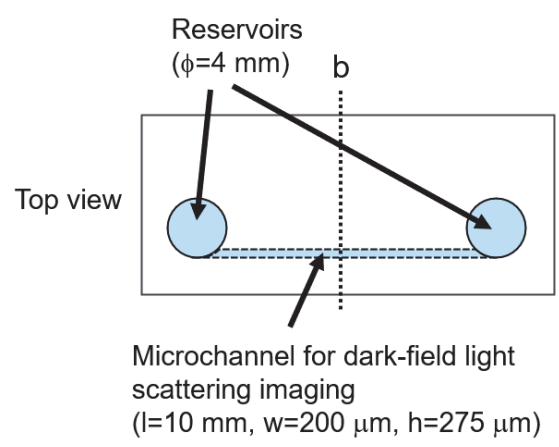

(b)

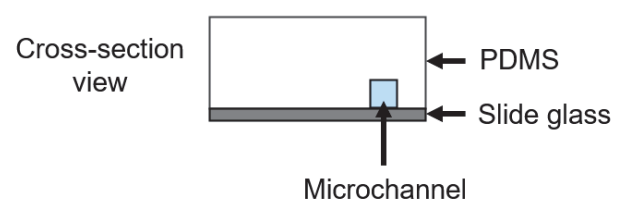

Fig. 1. (a) Schematics of the microfluidic chip and (b) cross-sectional image of the microfluidic chip.

\subsection{Master mold fabrication}

A silicon wafer (3-inch wafer, Canosis Co., Ltd., Tokyo, Japan) was spin-coated with a photoresist (SU-8 2100, Microchem, MA, USA) using the spin coater. The wafer was spun at a spin speed of 1500 rpm for $30 \mathrm{sec}$. Next, the wafer was soft baked at $65{ }^{\circ} \mathrm{C}$ for $5 \mathrm{~min}$ and then at $95^{\circ} \mathrm{C}$ for $30 \mathrm{~min}$ using a hot plate. The spin-coated wafer was able to be written using the photomask and a mask aligner (Union Double-View Mask Aligner PEM-800, Union Optical Co., Ltd., Japan) where the wafer was exposed at a dose of $1038 \mathrm{~mJ} / \mathrm{cm}^{2}$. Post-exposure, the wafer was baked at $65{ }^{\circ} \mathrm{C}$ for $5 \mathrm{~min}$ and then at $95^{\circ} \mathrm{C}$ for $15 \mathrm{~min}$, and was subsequently developed for $15 \mathrm{~min}$ in a developer solution (SU-8 Developer, MicroChem, MA, USA) and rinsed using isopropyl alcohol (2-Propanol, Kanto Chemical Co., Inc., Japan). Finally, the wafer was treated with trichloro $(1 \mathrm{H}, 1 \mathrm{H}, 2 \mathrm{H}, 2 \mathrm{H}-$ perfluorooctyl)silane $(97 \%$, SigmaAldrich Co., LLC., Japan) and left overnight in a desiccator under vacuum, to create the master mold used for the fabrication of the PDMS microchannel.

\subsection{Microchannel fabrication}

The schematic of the microchannel for dark-field imaging with light sheet illumination is illustrated in Fig. 1(a), in which two reservoirs of $4 \mathrm{~mm}$ diameter are shown and the microchannel was 10 


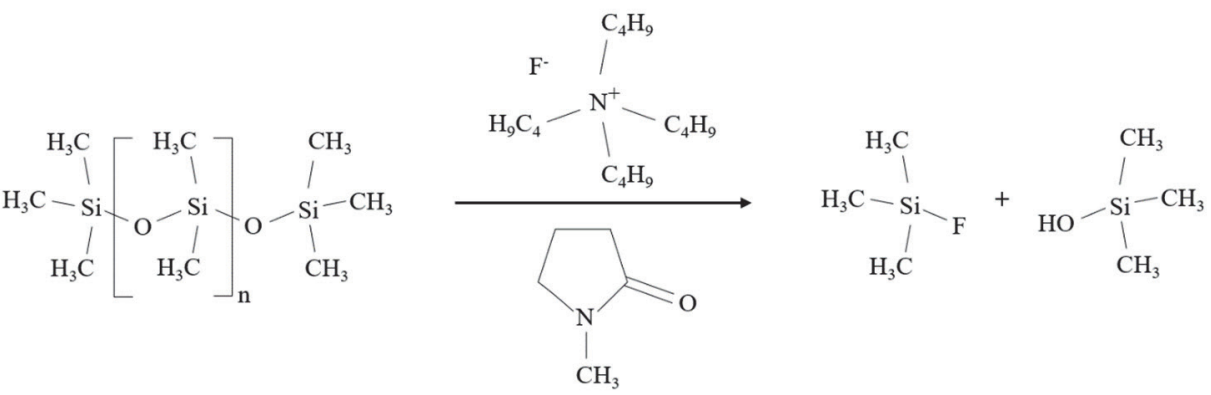

Fig. 2. Reaction scheme between TBAF and PDMS.

mm long, $200 \mu \mathrm{m}$ wide and $275 \mu \mathrm{m}$ thick. Figure 1(b) shows the cross-sectional view of the device. PDMS prepolymer (SYLGARD ${ }^{\mathrm{TM}} 184$ Silicone Elastomer Kit, Dow Inc., Japan) was used to make the PDMS device at a 10:1 ratio of base to curing agent. The PDMS solution was vortexed (Digital Vortex-Genie 2, Scientific Industries, Inc., USA) for 15 min to ensure thorough mixing. Next, the PDMS solution was poured over the master mold which was placed on a dish-shaped aluminum foil. To remove the air bubbles, the setup was placed in a vacuum desiccator under vacuum for $40 \mathrm{~min}$, after which the setup was cured at $150{ }^{\circ} \mathrm{C}$ for $10 \mathrm{~min}$. In sequel to this, the cured PDMS layer was peeled off from the master mold and a biopsy punch (Disposable Biopsy Punch 4 mm, Kai Industries Co., Ltd., Japan) was used to poke in the holes of the reservoirs. Next, a cutting tool (OLFA XSR-200, OLFA Corporation, Japan) was used to cut the PDMS microchannels into shape. The fabricated PDMS device was bonded onto a glass slide (Matsunami Micro Slide Glass, Matsunami Glass Ind., Ltd., Japan). A widespread method of bonding PDMS onto another substrate like glass involves exposing the PDMS to oxygen plasma $[29,30]$. However, after exposure, the bonding process must be completed promptly under one minute to obtain a strong bond [31], which can be difficult to do for bonding several different devices in quick succession and with accurate alignment. In bonding PDMS to glass, it has been detailed that the PDMS curing agent can be used as an intermediate layer between the PDMS and glass to form a lasting bond [32]. This method was utilized in this paper as it allows enough time to carefully place PDMS onto the glass for its desired alignment, without worry about time constraints. This easily allows for a batch of devices to be made at once. To achieve this, the silicon wafer was spin coated with the PDMS curing agent at a speed of $6000 \mathrm{rpm}$ for $30 \mathrm{sec}$. A stamp and paste method was used where the fabricated PDMS microchannel was stamped onto the spin coated silicon wafer and then pasted onto a glass slide. Then, the device was cured at $150{ }^{\circ} \mathrm{C}$ for $10 \mathrm{~min}$ to form the fabricated microchannel device.

\subsection{Etching test}

Firstly, the unetched microchannel was observed under a microscope (Nikon Eclipse L150, Nikon Corporation, Japan) with a camera (EO-5012C LE 62843, Edmund Optics Inc., USA) and software (uEye Cockpit, IDS Imaging Development Systems $\mathrm{GmbH}$, Germany) where images of the microchannel were collected. Next, the etchant solution of TBAF (Tetrabutylammonium fluoride solution $75 \mathrm{wt} \%$ in $\mathrm{H}_{2} \mathrm{O}$, Sigma-Aldrich Co., LLC., Japan) in the solvent NMP (1-methyl-2pyrrolidinone, $\geq 99 \%$, Sigma-Aldrich Co., LLC., Japan) was prepared. The solution was thoroughly mixed using a vortex mixer, and the etching test on the microchannel was subsequently carried out. The etchant solution was introduced into the left reservoir of the microchannel using a micropipette. To enable flow through the microchannel, a syringe ( $5 \mathrm{~mL}$ syringe, Terumo Corporation, Japan) was used as a vacuum on the right reservoir to pull in the solution as it reached the reservoir, and likewise, the microchannel was etched for $10 \mathrm{~min}$. The etched microchannel was observed under a microscope and images were collected, and were then analyzed using ImageJ (National Institute of Health, MD, USA). To end the test, the etchant was vacuumed out of the microchannel, following which the microchannel was dried to ensure that all the etchant had been removed. The etch distance was measured by calculating the difference in the width of the microchannel from before and after etching to obtain the etch distance. Subsequent etching tests involved varying the concentration of TBAF in the NMP solvent to obtain a range of etch distances of $0.10,0.40,0.68$, and $1.00 \mathrm{M}$. The reaction between TBAF and PDMS is described in Fig. 2. Based on prior research, the mechanism involves the fluoride ion from TBAF attacking the siloxane bond in 
PDMS in the polar solvent. Additionally, it has been found that the etch distance also depends on the polarity of the solvent and type of solvent which TBAF is present [26].

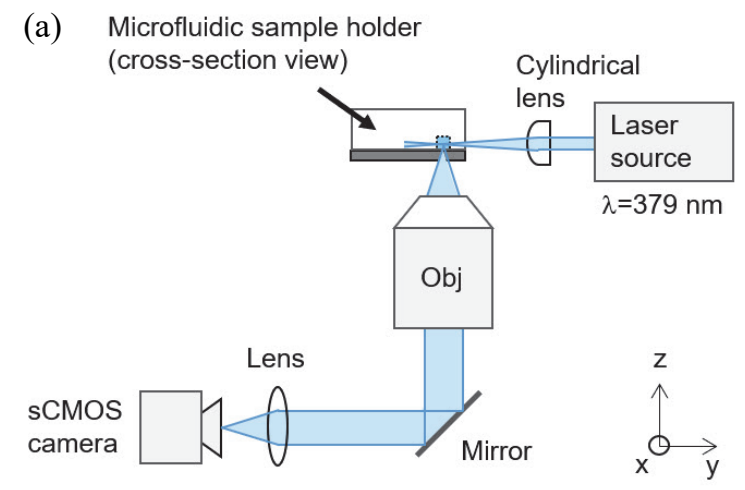

(b)

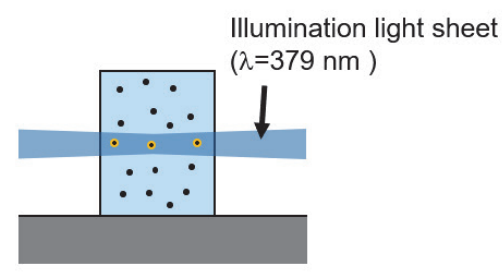

Fig. 3. Schematic of the optical setup for dark-field light scattering imaging. (a) The laser light was shaped as a sheet laser using a cylindrical lens and was focused inside the microchannel at the imaging plane. (b) Light-sheet illumination inside the microchannel at the image plane.

\subsection{SEM imaging}

Scanning Electron Microscope (FE-SEM Hitachi Regulus 8230, Hitachi High-Tech Corporation, Japan) was used to capture the crosssectional images of the unetched microchannel, and the microchannels etched for 1 and $10 \mathrm{~min}$. The microchannels were etched using a TBAF concentration of $0.68 \mathrm{M}$.

\subsection{Darkfield light scattering imaging}

In the light scattering test, standard solution of polystyrene nanoparticles (NIST Standard $100 \pm 6$ nm diameter Nanosphere ${ }^{\mathrm{TM}}$, Thermo Scientific, CA, USA) was diluted by a factor of $10^{6}$ with MilliQ that was filtered using a filter (Whatman ${ }^{\mathrm{TM}}$ Inorganic Membrane Filter Anotop ${ }^{\mathrm{TM}}$ 25, GE Healthcare Ltd., UK). Using a micropipette, the diluted nanoparticle solution was added into the reservoir of the PDMS microchannel and a syringe was used as a vacuum on the opposite reservoir to suck in and flow the nanoparticle solution into the microchannel. This process was performed for both unetched and etched microchannels. Subsequently, the nanoparticles were observed using a dark field light scattering imaging microscope. The optical setup portrayed in Fig. 3(a) is similar to prior research in which a cylindrical lens focuses on the shape of the laser $(\lambda=379 \mathrm{~nm})$ inside the microchannel $[18,19]$. Using the setup, light sheet illuminated nanoparticles within the imaging plane inside the microchannel is shown in Fig. 3(b).

\section{Results and discussion}

\subsection{Etch distance of PDMS microchannels}

The etch distances and concentrations are depicted in Fig. 4. The etch distance of PDMS with different concentrations of TBAF was evaluated to determine the concentration of the microchannel modification process. TBAF concentrations of 0.10 , $0.40,0.68$, and $1.00 \mathrm{M}$ in NMP solution were used and their average etch distances were evaluated to be $1.3 \pm 0.7,14.5 \pm 1.7,18.8 \pm 1.8$, and $17.7 \pm 1.2$ $\mu \mathrm{m}$, respectively, where overall the etch distance increases as the concentration of TBAF increases before it plateaus out. In previous research, similar results were observed, where the etch rate was measured and the concentration of TBAF was varied in solvents containing tetrahydrofuran and dimethylformamide [26]. As the concentration of TBAF increases, there was a greater amount of fluoride ions in the solution which can react with the siloxane bond, and thus the etching rate increased. This limitation can be considered as a result of the dissolution rate and diffusion speed of products of the etching reaction such as trimethyl silanol and fluoro-trimethyl silane. Based on this evaluation, TBAF at a concentration of $0.68 \mathrm{M}$ was recommended for use in further studies for the treatment of PDMS microchannels.

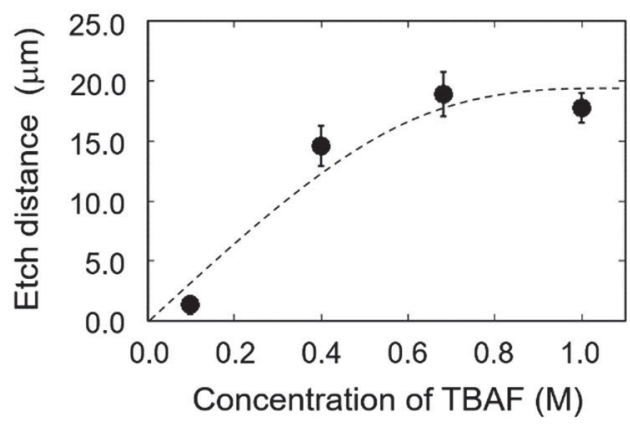

Fig. 4. Etch distance of PDMS with different concentration of TBAF in NMA solution $(0.10,0.40,0.68$, and $1.00 \mathrm{M}$ ) for $10 \mathrm{~min}$ treatment.

3.2. Effect of etching process to PDMS microchannel shapes

The effect of etching on the shapes of PDMS 
microchannel was investigated. For use in light sheet illumination, the microchannel wall should be placed in a vertical position. Thus, the cross-section images of the microchannels with and without etching treatment were obtained using SEM. Figure 5 shows the SEM images of the cross-section of the etched and unetched microchannels. Figure 5(a) shows the cross-sectional image of the microchannel without etching treatment. The microchannel has a rectangular shape of $200 \mu \mathrm{m}$ width and $275 \mu \mathrm{m}$ height. Figures 5(b) and (c) show the microchannel with etching treatment for 1 and 10 min, respectively. The shape of the microchannels and the verticality of the microchannel walls were preserved even after etching, as seen in the images of the etched microchannels (Figs. 5(b) and (c)). These results indicate that the etching process within $10 \mathrm{~min}$ with $0.68 \mathrm{M}$ TBAF will not degrade the light sheet illumination through the sidewall in darkfield light scattering imaging.

(a)

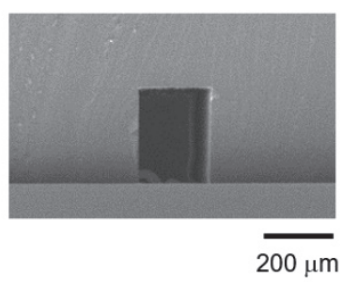

(b)

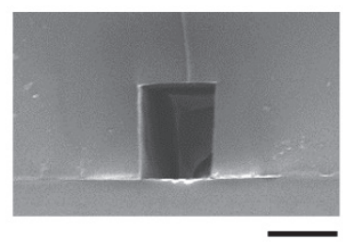

(c)

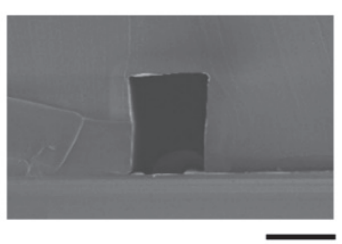

Fig. 5. Cross-sectional SEM image of the microchannels ( $w=200 \mu \mathrm{m}, h=275 \mu \mathrm{m}$ ). (a) Untreated, (b) TBAF treatment for $1 \mathrm{~min}$, and (c) TBAF treatment for $10 \mathrm{~min}$.

3.3. Darkfield light scattering imaging using the microchannel

The dark-field light scattering imaging was performed to observe the polystyrene nanoparticles which were flowed into the unetched and etched microchannels. A concentration of $0.68 \mathrm{M}$ TBAF was used to etch the microchannel for $10 \mathrm{~min}$. The light scattering images in the unetched and etched microchannels were shown in Figs. 6(a) and (b), respectively. In Fig. 6(a), light scattering was observed inside the microchannel near the microchannel wall of the light incident side, which gave a less clear image such that fewer nanoparticles were visible. This was likely due to the surface roughness of the PDMS microchannel as a result of the master mold that creates background noise that scatters the light in such a way that light sheet illumination is disturbed. Stripe patterns of scattered light were along with the incident light direction, and thus, those scattering can be considered as forward light scattering which is relatively strong compared with other directional scattering (Fig. 6(a)). In contrast, with the etching treatment of the microchannel, Fig. 6(b) shows that there was less light scattering since background noise was reduced, thereby obtaining a sharper image such that more nanoparticles were observable even near the microchannel wall.

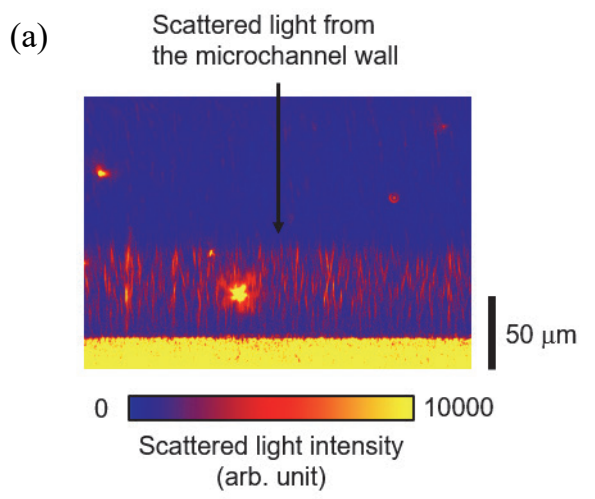

(b)

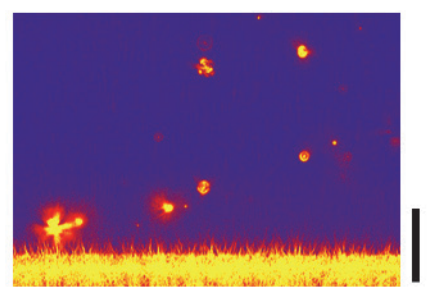

Fig. 6. Darkfield imaging of polystyrene nanoparticles $(\phi=100 \mathrm{~nm})$ with light sheet illumination. (a) Untreated microchannel and (b) microchannel with TBAF treatment.

\section{Conclusion}

The surface modification of the PDMS microchannel wall for dark field imaging was investigated using wet etching process. The etch distance of PDMS with $0.68 \mathrm{M}$ of TBAF in NMP solution was evaluated as $18.8 \pm 1.8 \mu \mathrm{m}$. Results 
from SEM analysis reveal that the rectangular shape and the vertical position of the microchannel wall were maintained through the etching treatment, and thus, was confirmed that the treated chip will not disturb the light sheet illumination. Dark-field light scattering imaging with light sheet illumination also showed the ability for observation of the individual polymer particles using the treated microchannels. This surface modification technique is expected to improve imaging quality and detection sensitivity by reducing the background noise resulting from the microchannel wall in optomicrofluidic applications accompanied by light sheet illumination.

\section{Acknowledgements}

This research was supported by Grants-in-Aid for Scientific Research (grant no. 15K21164 and 19K15416 to HT) from Japan Society for the Promotion of Science of Japan, and the Center of Innovation Program (COI STREAM), from Japan Science and Technology Agency (JST).

\section{References}

1. G. Zhou and S. C. Yao, Appl. Therm. Eng., 31 (2011) 228.

2. V. K. Natrajan and K. T. Christensen, Microfluid. Nanofluid., 9 (2010) 95.

3. V. K. Natrajan and K. T. Christensen, Exp. Fluids, 49 (2010) 1021.

4. Y. M. Kim, W. S. Kim, S. H. Lee, and J. Y. Baek, $20053 r d$ IEEE/EMBS Special Topic Conference on Microtechnology in Medicine and Biology, (2005) 292.

5. M. H. Farias, C. S. Castro, D. A. Garcia, and J. S. Henrique, J. Physics: Conf. Ser., 975 (2018) 12078.

6. S. R. Yadhuraj, S. Babu Gandla, S. S. Omprakash, B. G. Sudarshan, and S. C. Prasanna Kumar, Mater. Today Proc., 5 (2018) 21392.

7. B. Gorissen, C. Van Hoof, D. Reynaerts, and M. De Volder, Microsyst. Nanoeng., 2 (2016) 16045.

8. H. Wu, B. Huang, and R. N. Zare, Lab Chip, 5 (2005) 1393.

9. H. Takehara, A. Nagaoka, J. Noguchi, T. Akagi, T. Sakai, U. I. Chung, H. Kasai, and T. Ichiki, Biomicrofluidics, 7 (2013) 54109.

10. D. Uchida, M. Kanai, T. Nishimoto, and S. Shoji, IEEJ Trans. Sensors Micromachines, 124 (2004) 364.

11. S. Seethapathy and T. Górecki, Anal. Chim.
Acta, 750 (2012) 48.

12. A. Mata, A. J. Fleischman, and S. Roy, Biomed. Microdevices, 7 (2005) 281.

13. J. Huisken, J. Swoger, F. Del Bene, J. Wittbrodt, and E. H. K. Stelzer, Science, 305 (2004) 1007.

14. R. Regmi, K. Mohan, and P. P. Mondal, Microsc. Res. Tech., 76 (2013) 1101.

15. H. Deschout, K. Raemdonck, S. Stremersch, P. Maoddi, G. Mernier, P. Renaud, S. Jiguet, A. Hendrix, M. Bracke, R. V. den Broecke, M. Röding, M. Rudemo, J. Demeester, S. C. De Smedt, F. Strubbe, K. Neyts, and K. Braeckmans, Nanoscale, 6 (2014) 1741.

16. H. Jiang, T. Zhu, H. Zhang, J. Nie, Z. Guan, C. M. Ho, S. Liu, and P. Fei, Lab Chip, 17 (2017) 2193.

17. M. Lin, Q. Liu, C. Liu, X. Qiao, C. Shao, and X. Su, Biomed. Opt. Express, 9 (2018) 1692.

18. K. Kato, M. Kobayashi, N. Hanamura, T. Akagi, N. Kosaka, T. Ochiya, and T. Ichiki, Jpn. J. Appl. Phys., 52 (2013) 65.

19. T. Akagi, N. Hanamura, and T. Ichiki, $J$. Photopolym. Sci. Technol., 28 (2015) 727.

20. V. Majarikar, H. Takehara, and T. Ichiki, Microfluid. Nanofluid., 22 (2018) 110.

21. V. Majarikar, H. Takehara, and T. Ichiki, $J$. Photopolym. Sci. Technol., 32 (2019) 107.

22. J. Garra, T. Long, J. Currie, T. Schneider, R. White, and M. Paranjape, J. Vac. Sci. Technol. A, 20 (2002) 975.

23. B. Balakrisnan, S. Patil, and E. Smela, J. Micromech. Microeng., 19 (2009) 4.

24. J. N. Lee, C. Park, and G. M. Whitesides, Anal. Chem., 75 (2003) 6544.

25. Y. H. Nam, S. K. Lee, J. H. Kim, and J. H. Park, Microelectron. Eng., 215 (2019) 111008.

26. M. Kleiman, K. A. Ryu, and A. P. Esser-Kahn, Macromol. Chem. Phys., 217 (2016) 284.

27. R. Dahiya, G. Gottardi, and N. Laidani, Microelectron. Eng., 136 (2015) 57.

28. S. Gupta, A. Vilouras, and R. Dahiya, Microelectron. Eng., 221 (2020) 111157.

29. M. A. Eddings, M. A. Johnson, and B. K. Gale, J. Micromech. Microeng., 18 (2008) 6.

30. S. Bhattacharya, A. Datta, J. M. Berg, and S. Gangopadhyay, J. Microelectromech. Syst., 14 (2005) 590.

31. J. C. McDonald and G. M. Whitesides, Acc. Chem. Res., 35 (2002) 491.

32. B. Samel, M. K. Chowdhury, and G. Stemme, J. Micromech. Microeng., 17 (2007) 1710. 